\title{
Revisori dei papiri
}

or $=$ originale

$\mathrm{imm}=$ immagine a stampa o digitale

Galenus 1

Daniela Manetti

imm

Galenus 2

Daniela Manetti

imm

Herodotus 1

Elena Esposito

or

Herodotus 2

Elena Esposito

or

Herodotus 3

FaM

Herodotus 4

FaM/Raffaella Cribiore

imm

Herodotus 5

FaM

Herodotus 6

FaM/Chiara Meccariello

or

Herodotus 7

FaM/DC/Chiara Meccariello

imm

Herodotus 8

Elena Esposito

Or

or

$\mathrm{imm}$

Hesiodus 1

$\mathrm{MH}$

imm

Hesiodus 2

$\mathrm{MH}$

Hesiodus 3

$\mathrm{MH} / \mathrm{DC}$

Hesiodus 4

$\mathrm{MH}$

$\mathrm{imm}$

Hesiodus 5

$\mathrm{MH}$

Hesiodus 6

$\mathrm{MH} / \mathrm{GB}$

$\mathrm{imm} /$ or

$\mathrm{imm}$

$\mathrm{imm}$

$\mathrm{imm} /$ or

Hippocrates 1

Daniela Manetti

imm

Hippocrates 2

Daniela Manetti

imm

Hippocrates 3

Daniela Manetti

imm

Hippocrates 4

Daniela Manetti

imm

Hippocrates 5

Daniela Manetti

imm

Hippocrates 6

Daniela Manetti

$\mathrm{imm}$

Hippocrates 7

Daniela Manetti

imm

Hippocrates 8

Daniela Manetti

imm

Hippocrates 9

Daniela Manetti

imm

Hippocrates 10

Daniela Manetti

imm

Hippocrates 11

Daniela Manetti

imm 
Hippocrates 12

Daniela Manetti

imm

Hippocrates 13

Daniela Manetti

imm

Hippocrates 14

Daniela Manetti

imm

Hippocrates 15

Daniela Manetti

imm

Hippocrates 16

Daniela Manetti

imm

Hippocrates 17

Daniela Manetti

imm

Hippocrates 18

Daniela Manetti

imm

Hippocrates 19

Daniela Manetti

imm

Hipponax 1

Anika Nicolosi

imm

Hipponax 2

Anika Nicolosi

imm

Hipponax 3

Anika Nicolosi

imm

Hipponax 4

Anika Nicolosi

imm

Si ringraziano per la disponibilità in occasione dell'esame degli originali D. Obbink e D. Colomo (Sackler Library di Oxford). 